\title{
Myopotential inhibition of a bipolar pacemaker caused by electrode insulation defect
}

\author{
S. AMIKAM, H. PELEG, J. LEMER, AND E. RISS \\ From the Departments of Cardiology and Cardiac Surgery, Rambam Medical Center, \\ Aba Khoushy School of Medicine, Haifa, Israel
}

\begin{abstract}
A patient is described in whom myopotentials originating from the anterior abdominal wall muscles suppressed the implanted demand pacemaker despite its bipolar mode of action. This phenomenon was shown by simultaneous recording of the electrocardiogram and the electromyogram. At operation, a defect in the insulation of a previously repaired epicardial electrode was found lying in close proximity to these muscles. After repair of the insulation defect, normal pacemaker function was restored. It is suggested that the myopotentials leaked into the pacing system through the insulation defect, thereby suppressing the demand unit, which maintained its bipolar mode of pacing throughout.
\end{abstract}

It is now well established that potentials from skeletal muscles are capable of inhibiting demand unipolar pacemaker function (Wirtzfeld et al., 1972; Mymin et al., 1973; Ohm et al., 1974). The long single electrode configuration presents a large dipole to which myopotentials (Piller and Kennelly, 1974), as well as electromagnetic fields from the surrounding environment (Sowton et al., 1970), may be induced causing pacemaker inhibition. Bipolar demand systems are not prone to myopotential inhibition because the relative proximity of the electrodes gives a lower potential difference between the poles.

The purpose of this case report is to describe a patient in whom an insulation defect of one epicardial electrode allowed abdominal wall muscle potentials to suppress pacemaker function, despite the bipolar nature of the pacing system.

\section{Case report}

A 58-year-old woman had a permanent bipolar epicardial pacemaker implantation in 1965 for complete heart block and Adams-Stokes attacks. Since then the pacemaker generator has been electively replaced four times. The units had always been placed in the subcutaneous tissue of the anterior abdominal wall. During replacement of the fourth unit in 1973, one of the epicardial leads was erroneously transected and was immediately repaired. The repair was carried out by removing the insulation adjacent to the transected ends, intertwining the bared ends of the electrode coil, and reconstituting the insulation with a silicone adhesive-filled Silastic sleeve. The patient's subsequent course was uneventful and the pacemaker functioned well for a further 3 years, when a Medtronic demand bipolar model 5950 was electively implanted in June 1976. Four months later, the patient presented with signs of early erosion of the skin overlying the pacemaker unit. She underwent reimplanation of the same unit to a more medial position and in addition deep to the sheath of the rectus abdominis muscle.

On the day of operation, electrocardiographic monitoring showed periods of irregular pacing with lack of pacemaker spikes. Further analysis revealed that the pacemaker was intermittently inhibited, with the appearance of junctional escape beats (Fig. 1). This disturbance did not appear while the patient lay completely at rest and could be evoked again whenever the patient was asked to cough, to do a Valsalva manoeuvre, or to rise from a supine to a sitting position, i.e. while activating the abdominal wall muscles. However, the patient remained symptomless as she was resting in bed and because of the presence of escape beats which prevented long asystolic periods. Converting the pulse generator to the fixed-rate mode by external application of a magnet prevented this phenomenon during these exercises.

In order to demonstrate the mechanism of this interference, in addition to the electrocardiogram, 
an electromyogram from the left rectus abdominis muscle was simultaneously recorded during rest and during the above-mentioned manoeuvres (Fig. 2). It became evident that the pacemaker inhibition occurred only during rectus abdominis activation and was effectively corrected by the application of a magnet, changing the demand mode to a fixed-rate pacing.

On exposure at operation of the previously repaired epicardial electrode in the anterior abdominal wall, a defect was found in its insulation at the site of the repair. The proximal end of the Silastic sleeve surrounding the repair was not adherent and there was a small hole in the hardened silicone adhesive in which the repaired electrode wire was embedded. On squeezing the electrode, a small quantity of fluid escaped through this defect in the insulation. The hole was sealed with additional silicone adhesive, the original defective Silastic sleeve was repaired with adhesive and silk ligatures and finally a second Silastic sleeve was applied to surround the entire repair.

As a result of this procedure normal pacing was achieved, and since then suppression of pacing has not occurred either spontaneously or during abdominal wall muscle activation.

\section{Discussion}

Suppression of pacing in this patient occurred during abdominal wall muscle activation as shown by the simultaneous recording of the electrocardiogram and the electromyogram. Presumably the myopotentials were transmitted via the defect in the insulation of the electrode, which was in close proximity to the muscles of the anterior abdominal wall. This electrical activity was sensed by the demand unit and inhibited normal pacing. However, there appeared to be no outward escape of the pacing current through the defect in insulation, as had this occurred, twitching of the abdominal wall muscles would have been noted concomitant with pacemaker stimuli.

As mentioned previously, demand pacemaker units of the bipolar type are not subject to inhibition by myopotentials, this disturbance being strictly confined to the unipolar type. The occurrence of this type of malfunction in a bipolar demand pacemaker is most unusual. We were only able to find one published case report of an implanted bipolar demand pacemaker, in which pacing was suppressed during skeletal muscle activation. Widlansky and Zipes (1974) described a patient in whom isometric

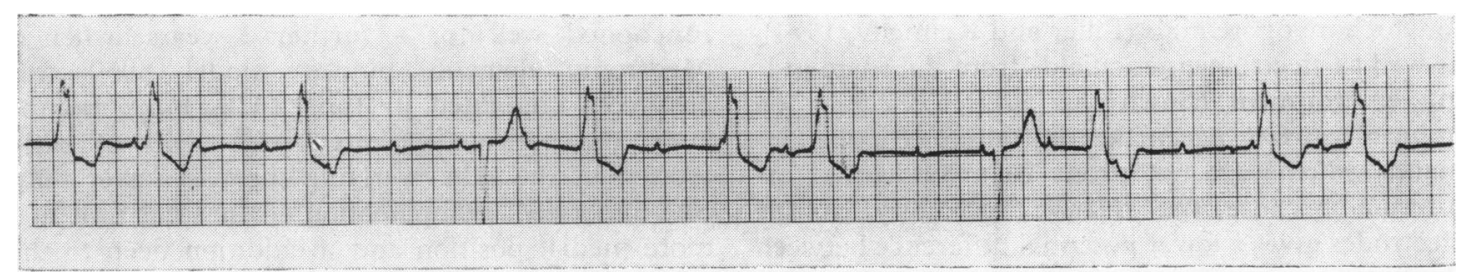

Fig. 1 Electrocardiographic monitor recording shows irregular pacing with short periods of ventricular asystole and junctional escape beats. Pacemaker spikes are not visible in this tracing.

EMG

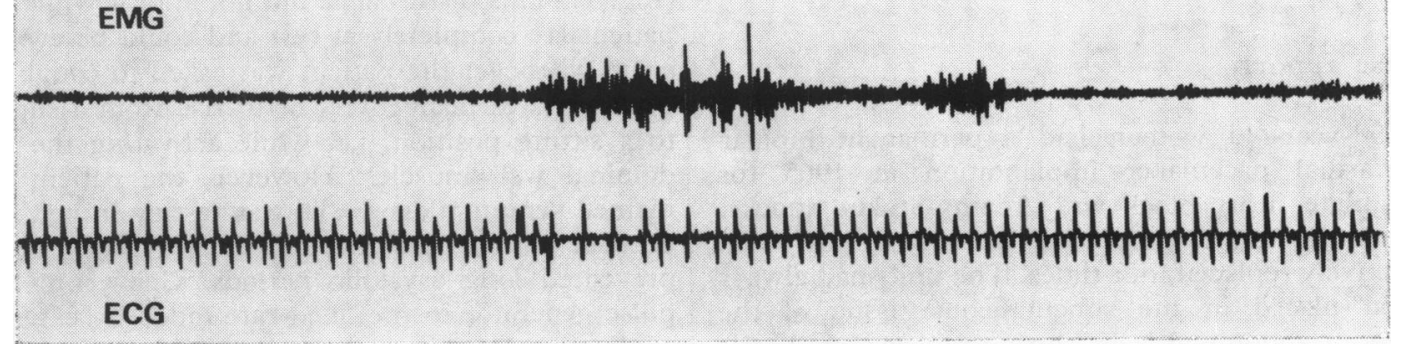

Fig. 2 Simultaneous recording of the electrocardiogram (lower tracing) and the electromyogram from the rectus abdominis muscle (upper tracing). Paper speed $5 \mathrm{~mm}$ per second. It is evident that the period of irregular pacing is concomitant with abdominal muscle activation. 
movements of the right pectoral muscle group totally suppressed bipolar demand pacemaker discharge. In their case, which had been paced by an endocardial electrode, it was discovered at operation that a proximal end of one of the electrodes was not correctly seated in the pulse-generator, and fluid had leaked into the connector boot. The authors suggested two possible explanations for the muscleinduced pulse-generator inhibition: (1) conversion of the bipolar demand system to a unipolar demand system by the fluid leak with subsequent sensing of myopotentials; and (2) generation of false (makebreak) electrical signals from repetitive interruption of the electrical circuit, caused by pectoral muscle activity, which were sensed by the demand unit, thereby suppressing its function. The authors tended to favour the second explanation as the pacemaker spikes remained unchanged before and after correction of the fault, indicating that unipolar pacing had not occurred during the period of malfunction.

The two possibilities cited cannot adequately explain the inhibition of pacing in our patient. We did not record on the electrocardiogram any change in the axis or amplitude of the pacemaker spike or in the paced QRS complexes during all stages of our patient's management. Therefore, conversion to a unipolar system did not occur. False (make-break) potentials could not have occurred in our patient, as electrode wire continuity was clearly shown along its entire length, both on $x$-ray film and at operation. We propose that the pacemaker inhibition in our patient was the result of a leakage of abdominal muscle myopotentials into the pacing system through the defect in the electrode insulation which was in close proximity to the muscles. This malfunction occurred without the conversion of the pacemaker system to a unipolar mode.

The authors wish to express their gratitude to Dr. V. Bialik of the Department of Orthopaedic Surgery, Rambam Medical Center, for the simultaneous electrocardiographic and electromyographic recordings.

\section{References}

Mymin, D., Cuddy, T. E., Sinha, S. N., and Winter, D. A. (1973). Inhibition of demand pacemakers by skeletal muscle potentials. Fournal of the American Medical Association, 223, 527-529.

Ohm, O. J., Bruland, H., Pedersen, O. M., and Waerness, E. (1974). Interference effect of myopotentials on function of unipolar demand pacemakers. British Heart fournal, 36, 77-84.

Piller, L. W., and Kennelly, B. M. (1974). Myopotential inhibition of demand pacemakers. Chest, 66, 418-420.

Sowton, E., Gray, K., and Preston, T. (1970). Electrical interference in non-competitive pacemakers. British Heart Fournal, 32, 626-632.

Widlansky, S., and Zipes, D. P. (1974). Suppression of a ventricular-inhibited bipolar pacemaker by skeletal muscle activity. Fournal of Electrocardiology, 7, 371-374.

Wirtzfeld, A., Lampadius, M., and Ruprecht, E. O. (1972). Unterdrückung von Demand-Schrittmachern durch Muskelpotentiale. Deutsche medizinische Wochenschrift, 97, 61-66.

Requests for reprints to Dr. S. Amikam, Department of Cardiology, Rambam Medical Center, Bat-Galim, Haifa, Israel. 